\title{
Spatially-resolved proteomics and transcriptomics: An emerging digital spatial profiling approach for tumor microenvironment
}

\author{
Nan Wang ${ }^{1}$, Rongshui Wang ${ }^{1}$, Xue Zhang ${ }^{1}, \mathrm{Xia} \mathrm{Li}^{1}$, Yan Liang ${ }^{2}$, and Zhiyong Ding ${ }^{1, *}$ \\ 1 Mills Institute for Personalized Cancer Care, Fynn Biotechnologies Ltd., Gangxing 3rd Rd, High-Tech and Innovation Zone, \\ Bldg. 2, Rm. 2201, Jinan City, Shandong Province 250101, PR China \\ 2 NanoString Technologies, Inc., Seattle, WA, USA
}

Received 17 September 2020, Accepted 4 December 2020, Published online 3 March 2021

\begin{abstract}
Digital spatial profiling (DSP) is an emerging powerful technology for proteomics and transcriptomics analyses in a spatially resolved manner for formalin-fixed paraffin-embedded (FFPE) samples developed by nanoString Technologies. DSP applies several advanced technologies, including high-throughput readout technologies (digital optical barcodes by nCounter instruments or next generation sequencing (NGS)), programmable digital micromirror device (DMD) technology, and microfluidic sampling technologies into traditional immunohistochemistry (IHC) and RNA in situ hybridization (ISH) approaches, creating an innovative tool for discovery, translational research, and clinical uses. Since its launch in 2019, DSP has been rapidly adopted, especially in immuno-oncology and tumor microenvironment research areas, and has revealed valuable information that was inaccessible before. In this article, we report the successful setup and validation of the first DSP technology platform in China. Both DSP spatial protein and RNA profiling approaches were validated using FFPE colorectal cancer tissues. Regions of interest (ROIs) were selected in the areas enriched with tumor cells, stroma/immune cells, or normal epithelial cells, and multiplex spatial profiling of both proteins and RNAs were performed. DSP spatial profiling data were processed and normalized accordingly, validating the high quality and consistency of the data. Unsupervised hierarchical clustering as well as principal component analysis (PCA) grouped tumor, stroma/immune cells, and normal epithelial cells into distinct clusters, indicating that the DSP approach effectively captured the spatially resolved proteomics and transcriptomics profiles of different compartments within the tumor microenvironment. In summary, the results confirmed the expected sensitivity and robustness of the DSP approach in profiling both proteins and RNAs in a spatially resolved manner. As a novel technology in highly complex spatial analyses, DSP endows refined analytical power from the tumor microenvironment perspective with the potential of scaling up to more analyzable targets at relatively low cell input levels. We expect that the DSP technology will greatly advance a wide range of biomedical research, especially in immuno-oncology and tumor microenvironment research areas.
\end{abstract}

Key words: Digital spatial profiling, Spatially resolved, Proteomics, Transcriptomics, Tumor microenvironment

\section{Introduction}

Since the paradigm shift to disentangle the underlying mechanisms of oncogenesis from solely tumor-centric principles to a more conceivably dynamic interplay and crosstalk involving normal and cancerous epithelial cells, fibroblasts, immune cells, and extracellular matrix (ECM) back in the mid-1980s, cumulative works were in progress to underpin the significant roles of the tumor microenvironment (TME) in localized neoplasm as well as a metastatic invasion [1]. Nowadays, research communities broadly appreciate that TME harbors complex populations of cell types distinctive from

\footnotetext{
*Corresponding author: zhiyong.ding@fynnbio.com
}

normal tissues. Dysfunction and reshaping of tumorsurrounding cell composition, networks, hypoxia, secretory fluids as well as extracellular $\mathrm{pH}$ all contribute to the formation of TME [2, 3].

Therapeutic implications derived from or hinting at TME related mechanisms have been brought to attention. Ample amounts of data manifested reciprocal interactions between TME and specific anticancer agents through direct intervention or indirect bypass effects on different cells within the microenvironment. By large, TME causes resistance to drugs by (1) inducing anti-apoptotic effects to compromise the efficacy of immunotherapies, (2) causing desmoplastic stroma which hampers penetration of chemotherapeutic agents to actionable sites, (3) prohibiting anticancer drug delivery through stalling 
blood supply, and (4) rendering constitutive activation of survival signaling pathways $[1,4,5]$. On the other hand, chemotherapy, radiotherapy, genotoxic agents, and anti-angiogenic drugs pose negative feedback effects on TME, many of which include reprogramming of immune cells, fibroblasts, and interstitial humoral regulation, involving inflammatory cytokines, tumor-initiating growth factors, and antibodies $[1,6]$. Therefore, it is not surprising from oncology perspectives that targeting tumor-TME interactions has become a research hotspot in the past 15 years and therapeutic interventions targeting tumorigenic TME have been extensively investigated. Early phase discoveries predominantly focused on oncogenic and angiogenic receptor tyrosine kinase (RTKs) such as epidermal growth factor receptor (EGFR), vascular endothelial growth factor receptor (VEGFR), and platelet-derived growth factor receptor (PDGFR). Expression of these RTKs on designated cells in regional and distal TME promotes tumor proliferation and vasculogenesis, which further strengthens the concept of mutual influence between evading cancer cells and surrounding TME [7-9].

With rapid development and advances in immunooncology, the past 10 years also witnessed an unprecedented magnitude in immunotherapies and combinational regimes tailored to obtain durable patient responses in various clinical studies. This in turn fundamentally redefined our ability to fight cancer. Two well-defined mainstream strategies to modulate immune responses in the context of TME have brought significant attention to date. Passive immunotherapy generally utilizes extrinsically administrated agents such as antibodies, antibodydrug conjugates (ADCs), tumor-infiltrating lymphocytes (TILs) or T cells subpopulations-based cell therapies (chimeric antigen receptor $\mathrm{T}$ cells, CAR- $\mathrm{T}$ or $\mathrm{T}$ cell receptor engineered $\mathrm{T}$ cells, TCR-T) to directly defeat cancer cells in a target-specific manner. On the contrary, active immunotherapy is designed to evoke endogenous immune activity via complex mechanisms [10]. While CAR-T based adoptive T cell therapy (ACT) is primarily designated to treat hematopoietic cancers and has shown its efficacy in some types of cancer such as diffuse large B-cell lymphoma (Yescarta) and non-Hodgkin lymphoma (Kymriah), it has not yet delivered largely convincing evidence for its applicability in patients with solid tumors [11]. In parallel, seeking for high affinity-enhanced, tumorreactive TCR-T while maintaining minimal levels of off-target toxicity in solid tumors still proved to be challenging [11]. In solid tumors, cancerous origins are often rooted in epithelial cells instead of immune cells, therefore there is no compensatory human immunoglobulin administration regime existing as is the case in B cell lymphoma therapies, making the solid tumors hard to manipulate. As a consequence, the lack of specificity is often accompanied by off-target toxicity leading to an array of downstream effects on T-cells and natural killer cells and causing cytokine release syndrome and other related immunopathogenic effects [12]. By nature, solid tumors also have limited trafficking to facilitate the regional spread of those therapeutic agents, which is even more important when treating metastatic cancer or cancer with multiple lesions at different organ sites. Moreover, histological heterogeneity and TME are key features prohibitive for drugs to function. Known causations include
$\mathrm{T}$ cell exhaustion upon predisposition to stimulatory antigens within confined microenvironments, which leads to restrained $\mathrm{T}$ cell activities and challenges in modulating common targetable neoantigens in different subsets of patients $[11,13$, 14]. As the opposite tactic, active immunotherapy adopts the major conceptual dogma to generate controlled and normalized immuno-active responses to circumvent undesirable immunogenic effects while maintaining expected on-target effects. This "modified" immune enhancement strategy opened up a new avenue to explore, especially with the successful underpinning of immune checkpoint inhibitor anti-PD-1/PDL1 therapies in an expended spectrum of late-stage malignancies. Many of those are solid tumors such as advanced melanoma, metastatic nonsmall cell lung cancer (NSCLC), metastatic squamous-cell head and neck carcinoma (HNSCC), hepatocellular carcinoma, renal cell carcinoma as well as others [15-20]. The remarkable success of this controlled immune activation further speaks to the profound but yet ill-defined mechanisms at local TME. This phenomenon was well supported by ample amount of evidence indicating that only measuring tumor biomarkers much as PD-L1 expression, tumor mutation burden (TMB), high microsatellite instability or mismatch repair deficiency (MSI-H)/dMMR) has limited predictive power to perfectly stratify patients [21-25].

Not until a few years ago did we realize that spatially defined phenotypic and molecular architectures may have eminent potentials to classify solid tumors according to their distinct characteristics. Three classes of TME outlined tumor types have been depicted: (1) "inflamed" tumor bears highly infiltrated immune cells and proinflammatory cytokines, (2) "immune desert" tumor bears minimal effector immune cell infiltrates, and (3) "immune excluded" tumor has immune cells present in the stroma but not the tumor parenchyma [26]. In line with this classification, by incorporating the TME information, the tumor immunity in the microenvironment (TIME) classification has been proposed. TIME emphasizes that resetting tumors into a TILs and PD-L1 positive status within the TME is critical to have pronounced immunogenic anti-tumor effects [27]. Those findings, though preliminary, uncovered a novel landscape that will set benchmarks for immune therapies in the future, and henceforth a deeper understanding of the TME and tumor interactions will be needed. To deconvolute mechanisms such as molecular causations of low TILs entry into tumor enriched regions, types of driver TILs components or cell populations to predict therapeutic responses and their underlying phenotypic and molecular pathways, the complexity and heterogeneity at a single tumor lesion within individual patients, as well as the acquired optimal synergies of immunotherapies combined with other conventional targeted therapies, radio(chemo)therapies, cancer vaccines, and oncolytic virus, more advanced technologies and platforms built for TME specific applications are in urgent demand [10, 28-30].

Tissue-based high-plex molecular profiling technologies emerged as one of the most prominent and powerful tools for TME based analysis due to their capacity to enable protein and RNA profiling while keeping spatial information intact. Traditional morphological driven low-to-medium plex protein assays such as multiplex IHC and cyclic immunofluorescence 
both rely on the cyclic collection of images and timeconsuming processes although their multiplexing capabilities are limited to 30-60 targets per slide [31-33]. Mass spectrometry (MS) based methods such as imaging mass spectrometry (IMS), though non-biased, require heavy instrumentation and pre-digestion as well as sophisticated setups to obtain the best spatial resolution down to $30 \mu \mathrm{m}$ [34-36]. Metal-isotopelabeled MS methods such as mass spectrometry immunohistochemistry (MSIHC) and multiplex ion-beam imaging (MIBI) also require expensive instrumentation and non-standardized laboratory procedures, and the plexing potential is plagued around 100 at maximum with semiquantitative measuring capacity $[35,37,38]$. From gene expression perspectives, relatively low plex technologies like RNAscope has outstanding sensitivity but cannot simultaneously profile hundreds of thousands of genes. More advanced spatial transcriptome technologies such as high-definition spatial transcriptomics (HDST), although capable of quantifying thousands of transcripts at even a sub-cellular resolution ( $2 \mu \mathrm{m}$ minimum at research level and $100 \mu \mathrm{m}$ at a commercial level in practice), are generally incompatible with formalin-fixed paraffin-embedded (FFPE) tissues, which are the most broadly available tissue type in clinical settings, and have expensive reagent costs that hamper general access for research purposes [39-41].

Digital spatial profiling (DSP) is a novel method for medium-to-high plex spatial profiling of proteins and RNAs compatible with FFPE samples [42]. It is developed by nanoString technologies, Seattle, WA, to empower quantification of proteins and RNAs under the same experimental setup, which is rather simplified with outstanding precision due to its bona fide molecular barcode counting technology proprietarily designed [43, 44]. One superior advantage of DSP over other technologies in the same category is the capacity to compartmentalize user-defined region of interests (ROIs) in spatialdirected patterns using multiple fluorescence labeling and in the meantime enabling more than 100-plex protein and 1,800-plex RNA analysis [42, 45, 46]. Using this technology, Dimm's group proved its robustness by comparing it with quantitative immunofluorescence (QIF) and by profiling 44 immune markers in pure compartments consisting of macrophage, leukocyte, and tumor respectively in a melanoma TMA cohort receiving checkpoint inhibitor therapies. They found the PD-L1 expression in the macrophage could potentially serve as a sole predictive biomarker for progression-free survival PFS, overall survival OS, and response [47]. Similarly in NSCLC patient cohorts, by pre-defined compartmentalization, they also identified a group of biomarkers that potentially play significant prognostic roles [48]. Moreover, two studies also used DSP to probe the spatially guided expression patterns of B lymphocytes within the tertiary lymphoid structures (TLSs) in the TME that are predictive of good patient responses. They found distinct B cell subsets in TME defined by higher proliferative capacity with higher Ki67 and CD40 expression, whereas pre-filtrating $\mathrm{T}$ cell populations within tumor areas have higher expression of TIM3, PD1, and GZMB indicative of poor response to immunotherapies $[49,50]$. These findings together strengthen the necessity of incorporating spatial information with highplex expression profiling, a new territory that is unexplorable using conventional methods, but one that will deepen our understanding of TME based research leading to the ultimate improvement of immunotherapies.

\section{Technical briefs of DSP}

DSP combines conventional immunofluorescence techniques with digital barcoded antibodies or oligonucleotides to allow morphologically outlined and high-plex expression profiling. The experimental procedure starts with routine $5 \mu \mathrm{m}$ FFPE or fresh-frozen tissue sections mounted on charged slides. A multi-color fluorescence staining mixture (up to 4) with additional high-plex probe sets (oligo-conjugated antibodies or target specific oligos) designed to suit experimental needs either for proteins or RNAs respectively is incubated with the slides (similar to conventional immunofluorescence or IHC). This one-step incubation is then followed by high-resolution scanning using the DSP instrument to allow ultimate precision for ROI selection. ROI selection accepts various user-defined contours from circle to any polygon shapes. The collection of digital probes within ROIs is performed by a micro-sized programmable digital micromirror device (DMD) which sheds UV light on selected ROIs to release the photocleavable oligos. Released oligo probes are aspirated via a capillary sipper sequentially. The resultant single strand oligos are then hybridized to target specific fluorescence reporter probes to enable digital counting performed by the nanoString nCounter digital barcode counting system, and the numerally transformed readings directly reflect the expression level of targets [44]. The schematic presentation of the DSP workflow is shown in Figure 1. The accompanying video demonstrates a visual protocol of the DSP approach (Video 1).

\section{Methods in brief}

\section{Validation of DSP performance with cell lines}

Two high-density homogenous HEK293 cell microarray slides obtained from nanoString were used to validate the DSP approach at Mills Institute for Personalized Cancer Care (MIPCC), Fynn Biotechnologies (FynnBio). The DSP core protein module (Immune Cell Core Profiling) consisting of 20 specific antibodies for immune-related targets and a standard morphology panel comprising Pan-Cytokeratin (Pan-CK, marker for epithelium cells/tumor cells), CD45 (marker for immune cells) and SYTO13 (nuclei) were used for the validation (for the full list of core protein targets and other details, refer to nanoString websites https://www.nanostring.com). Three circular ROIs on each slide spanning from the diameter range of $200 \mu \mathrm{m}, 400 \mu \mathrm{m}$, and $600 \mu \mathrm{m}$ were selected respectively, and subject to downstream DSP processes.

\section{Spatial profiling of protein and RNA of colorectal cancer tissue using DSP}

We used serial sections of a colorectal cancer FFPE tissue mounted on slides to validate the spatial profiling of both protein and RNA by the DSP approach. FFPE slides went through conventional tissue pre-processing (deparaffinization 


\section{Scan and imaging}
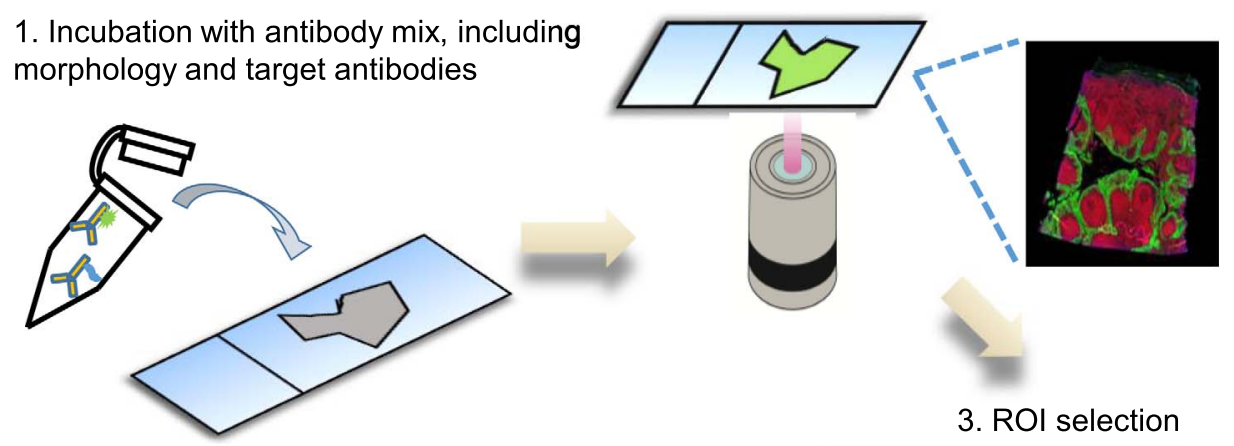

3. ROI selection

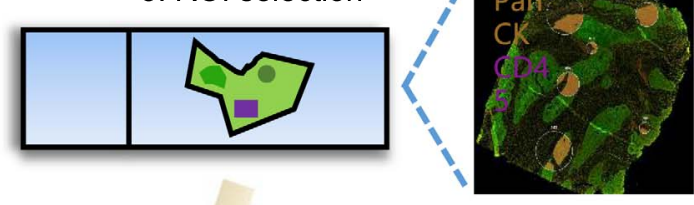

5. Digital counting and data analysis
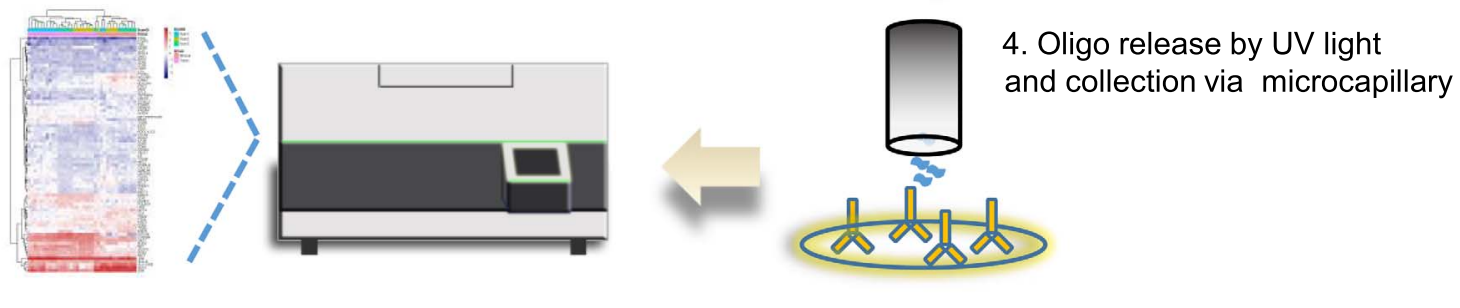

Figure 1. Schematic diagram of DSP workflow. 1. FFPE tissue sections mounted on slides are processed by standard IHC procedure and incubated with morphology marker reagents (fluorescence-labeled antibodies or dyes) and a mix of oligo conjugated antibodies. 2. Highquality images up to four channels of fluorescence are obtained. 3. ROIs are selected on the DSP instruments based on the morphological fluorescence marker. 4. Oligos in specific ROIs are released by UV light and collected by a microcapillary tube and dispensed into 96-well plates for subsequent processing. This oligo release and collection cycle is repeated for each ROI. 5. Digital counting by nCounter instruments to generate protein or RNA profiling data in a spatially resolved manner. Alternatively, highly multiplexed RNA profiling could be achieved using specific sequence adaptors (Unique Dual Index UDI) to allow NGS readout.

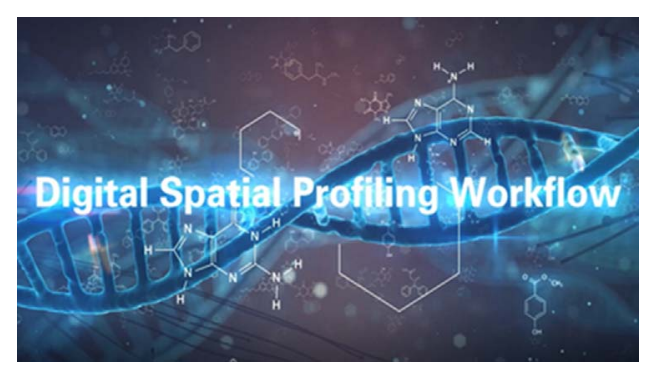

Video 1. A visual protocol of the Digital Spatial Profiling (DSP) Approach. https://vcm.edpsciences.org/10.1051/vcm/2020002\#V1

and rehydration). For protein profiling, 20-plex immune cell profiling core module plus 2 additional modules (IO Drug Target and Immune Cell Typing), each containing about 10 targets were used. For RNA profiling, the immune pathways module with a 96-plex RNA probe set was used. In both profiling approaches, we applied the same standard fluorescencelabeled morphology marker panel consisting of Pan-CK for epithelial and tumoral regions, CD45 for immune cells, and nuclear stain SYTO13 for tri-colored tissue compartmentalization as ROI selection references. Twelve circular ROIs on each slide were drawn and selected. For protein profiling, the areas of selected ROIs were between 20,000 and 120,000 $\mu^{2}$. For RNA profiling, due to the relatively low counts compared to proteins, ROIs were selected between 38,000 and $380,000 \mu \mathrm{m}^{2}$ to ensure successful quality controls in data analysis. All oligos from 48 ROIs for protein profiling and 48 ROIs for RNA profiling were collected into 96-well plates using the proprietary UV-guided technology in the DSP approach. Resultant oligos representing individual targets for individual ROIs were pooled together by column, hybridized to the beads-on-string barcoded counterpart oligos, and counted by nanoString nCounter.

\section{Data processing and statistical analysis}

Raw reporter code count (RCC) files generated by nCounter were transformed into tabulated excel files with all interpretable values presented for all ROIs. External RNA 
A

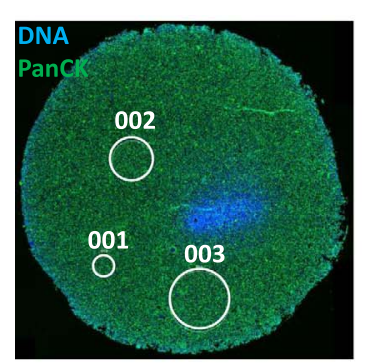

Slide 1

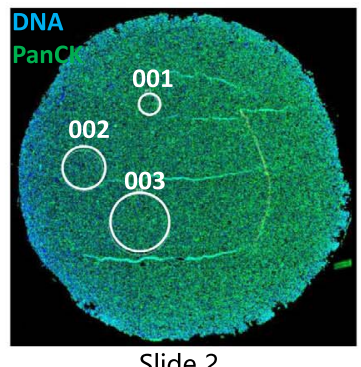

Slide 2
C
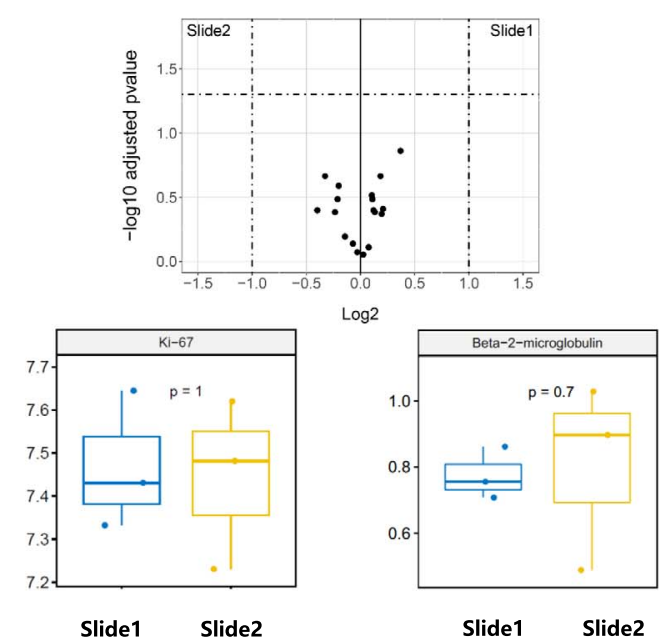

B
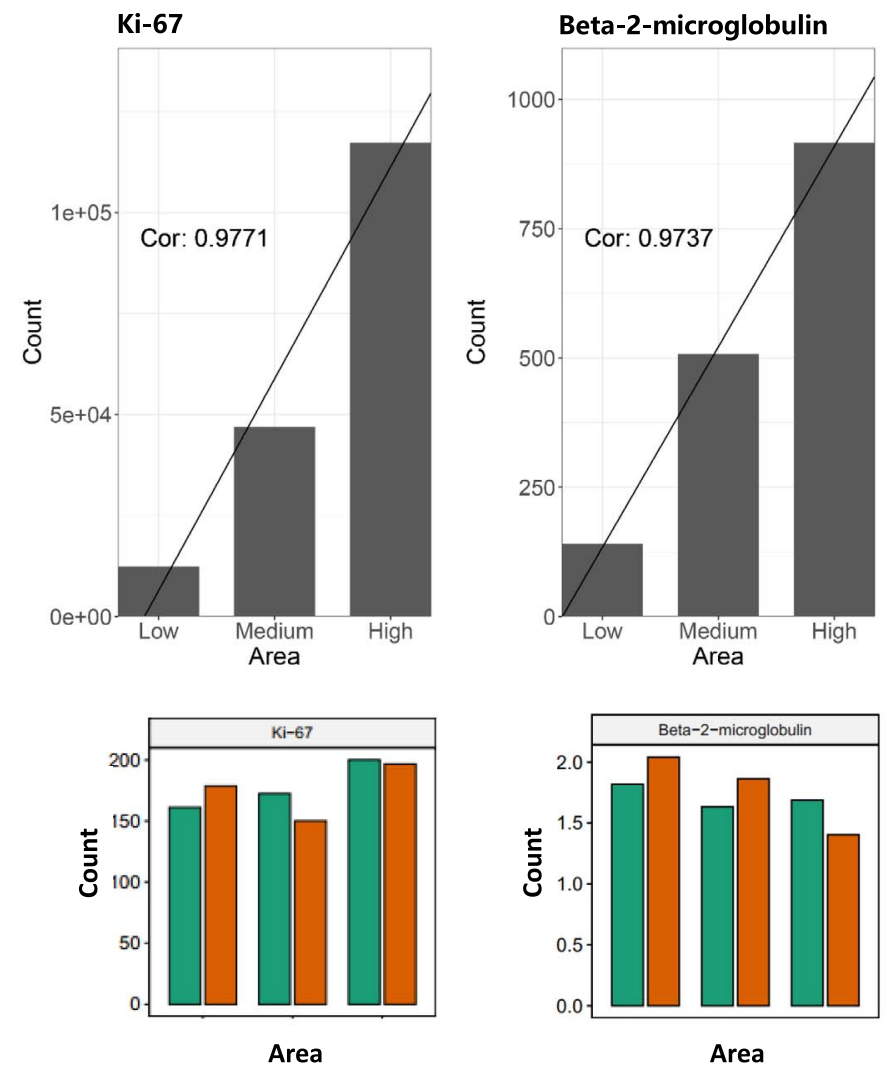

Figure 2. Validation of the DSP protein profiling workflow. (A) ROI selection of FFPE HEK293 cell pellet slides. Circular ROIs of three different sizes $(200 \mu \mathrm{m}, 400 \mu \mathrm{m}$, or $600 \mu \mathrm{m}$ in diameter) were selected on each slide, labeled as 001, 002, and 003, respectively. Cell nuclei were stained by STYO13 (blue) and cells were stained by fluorophore-labeled Pan-CK antibody (green). (B) Upper panel: Correlation of nCounter counts ( $y$-axis) and ROI area ( $x$-axis). Lower panel: nCounter counts of Ki-67 and Beta-2 microglobulin from three ROIs of different sizes normalized with housekeeping genes in duplicate slides (green and orange). (C) Volcano plot and box plots of individual proteins in HEK293 cells from duplicate slides. $\operatorname{LogFC}>1$ and $p$-value $<0.05$ are denoted as significant. Data are FDR adjusted with blue and yellow representing individual slides.

Controls Consortium (ERCC)) internal spike-in negative and positive controls were used to assess data quality and the performance of each experiment. Based on the values of ERCC normalization factors, a range between 0.3 and 3 for individual ROIs was chosen to eliminate outliers that are incompatible for downstream analysis. To filter out any skewness of the data, minimum nuclei count of 20 and surface area of $1,600 \mu \mathrm{m}^{2}$ were set for protein profiling and minimum nuclei count of 200 and surface area of $6,000 \mu \mathrm{m}^{2}$ for RNA profiling. Data that pass quality control (QC) were then normalized by the geometric mean of selected housekeeping gene expression counts. UBB, RAB7A, OAZ1, POLR2A, and SDHA were used to normalize nCounter counts for RNA profiling. S6, Histone $\mathrm{H} 3$, and GAPDH were used to normalize for protein profiling. Normalized data were then log-transformed and subject to further analysis. For statistical analyses, paired $t$-tests (intraslide ROIs) and unpaired $t$-tests (inter-slide ROIs) were used to test differential expression profiles. False discovery rate (FDR) was adjusted using the Benjamini-Hochberg method and all $p$ values were set to 0.05 as the cutoff. For unsupervised hierarchical clustering, log transformed data were median-centered before plotting. All unsupervised clustering and principal component analysis (PCA), statistical calculations, and all graphical presentations were conducted using $\mathrm{R}$ graphical user interfaces (GUIs).

\section{Results of DSP workflow validation}

Two FFPE HEK293 cell pellet slides were used at MIPCC to validate the DSP protein profiling workflow, with the core protein module consisting of 20 antibodies, together with IO Drug Target module and Immune Cell Typing module (38 antibodies in total). The quantification capability of the approach was assessed by measuring the readouts generated from three ROIs at different sizes $(200 \mu \mathrm{m}, 400 \mu \mathrm{m}$, and $600 \mu \mathrm{m}$ in diameter) (Fig. 2A). For proteins with known expression in HEK293 cells, nCounter counts from ROIs with different sizes showed a linear increase when plotted against the area of 
A

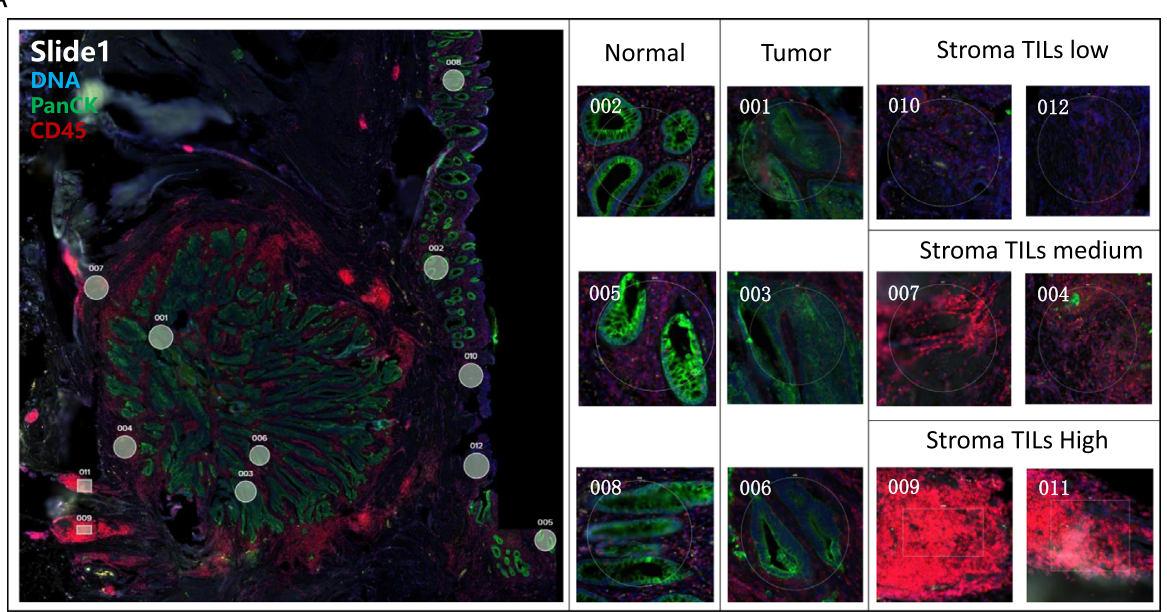

B
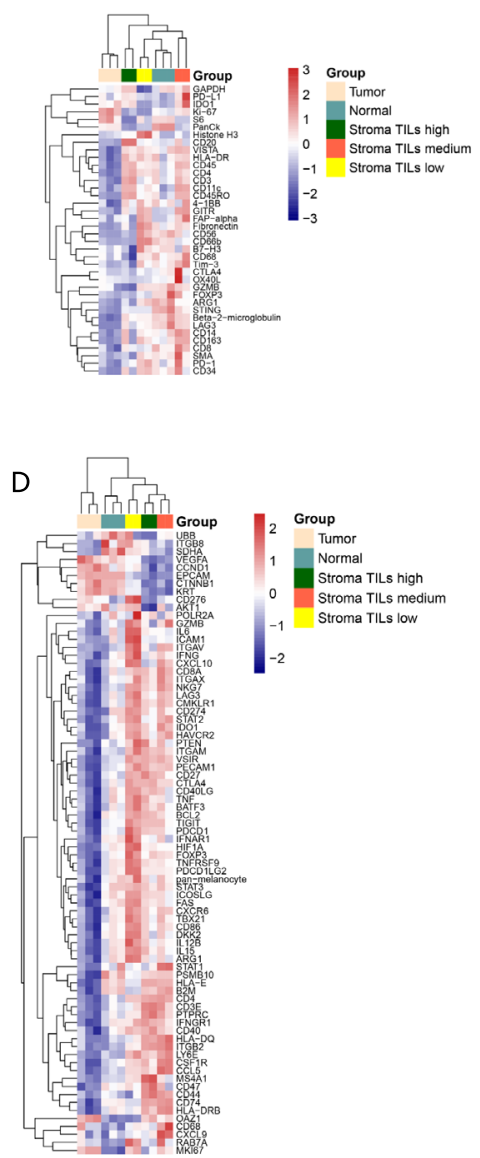

C

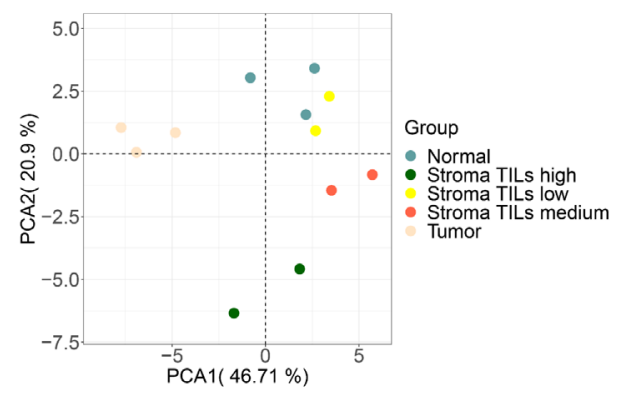

$E$

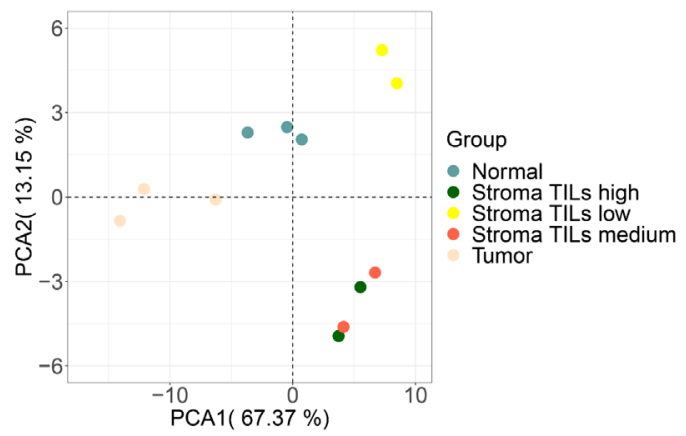

Figure 3. Validation of the DSP protein and RNA profiling approaches using colorectal cancer tissues. (A) ROI selection of normal, tumor, and stromal cells on FFPE tissue slides. Morphology markers (SYTO13 for nuclei staining, blue; Pan-CK for tumor/ epithelial cells, green; CD45 for immune cells, red) were used to guide 12 ROI selections of tumor, stroma, or normal epithelial cells enriched areas. Based on the abundance of tumor-infiltrating lymphocytes (TILs), 2 stromal TILs low, 2 stroma TILs medium, and 2 stroma TILs high ROIs were selected. Two sets of ROIs at the same locations were selected from 2 serial sections of the FFPE tissue for protein and RNA profiling, respectively. (B) Unsupervised hierarchical clustering of 12 ROIs representing different histological regions (normal epithelial cells, tumor, and stroma) based on 38-plex protein profiling. ROIs belonging to the same group are color-coded. (C) PCA clustering of different group assignments in protein profiling. Groups represents ROIs from different histological regions. (D) Unsupervised hierarchical clustering of 12 ROIs representing different histological regions (normal epithelial cells, tumor, and stroma) based on 78-plex RNA profiling. ROIs belonging to the same group are color-coded. (E) PCA clustering of different group assignments in RNA profiling. Groups represent ROIs from different histological regions. 
the ROIs. Two representative proteins (high and medium count values), Ki-67 (Cor $=0.9771)$ and Beta-2 microglobulin $($ Cor $=0.9737)$ are shown in Figure 2B. Upon housekeeping gene normalization using S6, Histone H3, and GAPDH, the size-oriented effects were removed allowing direct inter-ROI comparison of protein expression, which in this case had no statistically significant difference as expected (Fig. 2B, lower panel). On this basis, by pooling normalized counts from three ROIs within each slide, no protein was differentially expressed between the duplicated slides under $\log 2$ fold-change (Log FC) $>1$ and adjusted $p$-value $<0.05$ as cutoffs (Fig. 2C). Taken together, our data proved the reliability of the DSP technology and ensured our follow-up studies on tissue-based assays.

\section{Validation of DSP on colorectal cancer tissues}

To gain further insight into the validity of DSP on tissuebased assays, we used two serial sections of a colorectal cancer FFPE tissue to allow protein and RNA profiling in parallel to directly compare expression at transcript and protein levels. To generate biologically meaningful data, we selected 12 ROIs on each slide at different histological regions according to fluorescence-guided staining and annotated as different groups for clustering analysis. Representative scans and ROIs selected are graphically demonstrated in Figure 3A. We first carried out ROI selection for RNA profiling and then cross-referenced them to all ROIs for the protein profiling and this ensured a direct in-situ comparison between RNA and protein expression at this sub-histological level. For both protein and RNA profiling, unsupervised hierarchical clustering assigned tumor, stroma, and normal epithelial cells into distinct groups. (Figs. 3B and 3D). In addition, stroma ROIs with a different abundance of tumor-infiltrating lymphocytes (TILs) were also effectively clustered. These clearly defined clusters were also reflected by the PCA data with clearly separated tumoral and stromal groups (Figs. 3C and 3E). Within the stroma compartment, as our ROI selection criteria were primarily based on immune cell-enriched regions, our data demonstrated a great concordance as indicated by high CD4, CD3, and CD8 expression at the protein level and CD4 and CD8A at the RNA level. Again, higher Pan-Ck protein expressions were observed at both tumor and normal epithelial regions whereas tumor regions featured relatively higher Ki-67 and AKT indicating high proliferating potentials. Moreover, normal epithelial regions had generally higher effective infiltrated immune cells as compared to corresponding tumor regions as indicated by the expression of Granzyme B and CD8 at the protein level (Fig. 3D). Those findings together reconfirmed the robustness of this assay.

\section{Discussion}

To validate the DSP technology platform, we performed DSP spatial profiling for both protein and RNA using FFPE colorectal cancer tissues mounted on slides. Regions of Interest (ROIs) were selected in the areas enriched with tumor cells, stroma/immune cells, or normal epithelial cells on serial sections of the tissue to compare protein data with RNA data. Currently, no parallel technology could facilitate proteogenomic characterization at this sub-histological resolution. The results showed that the DSP spatial profiling data were of high quality and consistent after standard normalization and process. Results of further analyses including unsupervised hierarchical clustering and PCA demonstrated that ROIs from the tumor, stroma/ immune cells, and normal epithelial cells were grouped into distinct clusters, indicating that the specific protein or RNA expression information from different cellular contexts on the slides was precisely captured. Though our demonstrative work is simple, many other sophisticated approaches of DSP usage have been demonstrated elsewhere [42, 51-53]. Several ROI selection strategies are available to suit various research needs such as global deep profiling for full tissue sections, segmentation mode for precise analysis between tissue compartments, and rare cell profiling mode [42]. In our experience, even at a low cell input level, DSP could still perform well but our work is currently limited to cell line data with more to elucidate at the tissue level and that could hopefully inform us more on the technical robustness and multiplexing capacity in the future. Our tissue work aimed to assess the reliability of the assay and by profiling only 12 ROIs with very few replicates within each group, we were able to show the very clear separation of predefined groups (normal, tumor-enriched, and immune-enriched regions) based on both transcript and protein levels proving the assay reliability.

The latest development of DSP is to couple with NGS and thus to expand the multiplexing capabilities for RNA profiling [42]. Currently, commercially available detectable targets are around 100 for proteins and 1,800 for RNAs covering comprehensive key genes in oncology, immune-oncology, as well as many other pathways in cancer. Future development to cover the full gene expression spectrum is underway.

As an innovative research tool, DSP holds multiple attributes that are unmet by many parallel technologies. First, accredited by its proprietary barcode technology, it is applicable to measuring both proteins and RNA in-situ in a highly quantitative manner. We have tested in our hand the quantification capability of DSP for proteogenomic analysis and both levels have shown reliable data respectively. Secondly, this is one of the very few technologies dedicated to high-plex FFPE samples profiling at sub-spatial resolution and this significantly broadens the application potential of the technology [46]. Thirdly, customizable fluorescence-guided morphology markers allow computationally defined segmentation within ROIs facilitating further purification of samples and/or rare cells to be analyzed at user's choices. Lastly, unlike laser captured microdissection (LCM), samples remain intact during the entire workflow allowing other downstream application of samples such as IHC and conventional HE staining. When the slides are available, HE staining is recommended to provide additional information. On the contrary, DSP also bears certain limitations. Its resolution cannot reach the single-cell level though computational-based inference tools may be applied in the near future based on bulk transcription profiling data [54]. Though near single-cell sensitivity for some specific protein was achieved by others and in our hands (data not shown), a better signal-noise ratio for certain antibody probes may need to be improved, [42]. Currently, an alternative method is to select multiple rare cells containing ROIs a user-defined 
phenotype group to obtain sufficient counting. Expansion of protein targets is also essential for higher plexing needs. Moreover, the future direction of integrating DSP with other spatial analysis software may help obtain multi-dimensional data for advanced analysis [54].

\section{Conclusion}

DSP developed by nanoString, is a groundbreaking technology providing insights into a wide range of biomedical research, especially immuno-oncology and tumor microenvironment areas. DSP integrates multiple cutting-edge technologies, including high-throughput detection technologies (NGS or digital optical barcodes), programmable DMD technologies, and microfluidic sampling, into traditional IHC and RNA in situ hybridization (ISH) approaches, creating an innovative tool for spatial-resolved proteomics and transcriptomics analyses on FFPE samples as well as fresh frozen tissues. We set up the first DSP platform in China at MIPCC, FynnBio in 2019, right after nanoString Technologies launched the technology. As an early method establishment approach, we have validated this innovative technology platform demonstrating the expected sensitivity and robustness of the approach. We expect that the DSP platform will greatly advance biomedical research especially in immuno-oncology and tumor microenvironment areas.

Though the limitation of numbers of cells in selected ROIs, discrete cells labeled by specific morphology marker could still be collected and counted sufficiently. Currently, up to 2,000 RNAs could be detected for each ROI because of the replacement of the readout method, and panels for the entire transcriptome or even genome could go into service in the foreseeable future.

\section{Conflict of interest}

The authors declare that they have no conflict of interest.

\section{References}

1. Maman S, Witz IP. A history of exploring cancer in context. Nat Rev Cancer. 2018;18(6):359-76.

2. Joyce JA, Pollard JW. Microenvironmental regulation of metastasis. Nat Rev Cancer. 2009;9(4):239-52.

3. Ghirelli C, Hagemann T. Targeting immunosuppression for cancer therapy. J Clin Invest. 2013;123(6):2355-7.

4. Kroemer G, Galluzzi L, Kepp O, et al. Immunogenic cell death in cancer therapy. Annu Rev Immunol. 2013;31:51-72.

5. Shiao SL, Coussens LM. The tumor-immune microenvironment and response to radiation therapy. J Mammary Gland Biol Neoplasia. 2010;15(4):411-21.

6. Katz OB, Shaked Y. Host effects contributing to cancer therapy resistance. Drug Resist Updat. 2015;19:33-42.

7. Pegram M, Slamon D. Biological rationale for HER2/neu (c-erbB2) as a target for monoclonal antibody therapy. Semin Oncol. 2000;27(5 Suppl 9):13-9.

8. Jain RK. Normalizing tumor microenvironment to treat cancer: bench to bedside to biomarkers. J Clin Oncol. 2013;31(17): 2205-18.
9. Nguyen AN, Stebbins EG, Henson M, et al. Normalizing the bone marrow microenvironment with $\mathrm{p} 38$ inhibitor reduces multiple myeloma cell proliferation and adhesion and suppresses osteoclast formation. Exp Cell Res. 2006;312(10):1909-23.

10. Sanmamed MF, Chen L. A paradigm shift in cancer immunotherapy: From enhancement to normalization. Cell. 2019;176(3):677.

11. Weber EW, Maus MV, Mackall CL. The emerging landscape of immune cell therapies. Cell. 2020;181(1):46-62.

12. Kennedy LB, Salama AKS. A review of cancer immunotherapy toxicity. CA Cancer J Clin. 2020;70(2):86-104.

13. Wherry EJ, Blattman JN, Murali-Krishna K, et al. Viral persistence alters CD8 T-cell immunodominance and tissue distribution and results in distinct stages of functional impairment. J Virol. 2003;77(8):4911-27.

14. Schumacher TN, Schreiber RD. Neoantigens in cancer immunotherapy. Science. 2015;348(6230):69-74.

15. Forde PM, Chaft JE, Smith KN, et al. Neoadjuvant PD-1 Blockade in resectable lung cancer. N Engl J Med. 2018;378(21): 1976-86.

16. Ferris RL, Blumenschein G Jr, Fayette J, et al. Nivolumab for recurrent squamous-cell carcinoma of the head and neck. N Engl J Med. 2016;375(19):1856-67.

17. Kang YK, Boku N, Satoh T, et al. Nivolumab in patients with advanced gastric or gastro-oesophageal junction cancer refractory to, or intolerant of, at least two previous chemotherapy regimens (ONO-4538-12, ATTRACTION-2): a randomised, double-blind, placebo-controlled, phase 3 trial. Lancet. 2017;390(10111): 2461-71.

18. Schachter J, Ribas A, Long GV, et al. Pembrolizumab versus ipilimumab for advanced melanoma: final overall survival results of a multicentre, randomised, open-label phase 3 study (KEYNOTE-006). Lancet. 2017;390(10105):1853-62.

19. El-Khoueiry AB, Sangro B, Yau T, et al. Nivolumab in patients with advanced hepatocellular carcinoma (CheckMate 040): an open-label, non-comparative, phase 1/2 dose escalation and expansion trial. Lancet. 2017;389(10088):2492-502.

20. Ribas A, Wolchok JD. Cancer immunotherapy using checkpoint blockade. Science. 2018;359(6382):1350-5.

21. Yarchoan M, Hopkins A, Jaffee EM. Tumor mutational burden and response rate to PD-1 inhibition. N Engl J Med. 2017; 377(25):2500-1.

22. Lu S, Stein JE, Rimm DL, et al. Comparison of biomarker modalities for predicting response to PD-1/PD-L1 checkpoint blockade: A systematic review and meta-analysis. JAMA Oncol. 2019;5(8):1195-1204.

23. Tumeh PC, Harview CL, Yearley JH, et al. PD-1 blockade induces responses by inhibiting adaptive immune resistance. Nature. 2014;515(7528):568-71.

24. Spranger S, Luke JJ, Bao R, et al. Density of immunogenic antigens does not explain the presence or absence of the T-cellinflamed tumor microenvironment in melanoma. Proc Natl Acad Sci USA. 2016;113(48):E7759-E68.

25. Danilova L, Wang H, Sunshine J, et al. Association of PD-1/ PD-L axis expression with cytolytic activity, mutational load, and prognosis in melanoma and other solid tumors. Proc Natl Acad Sci USA. 2016;113(48):E7769-E77.

26. Chen DS, Mellman I. Elements of cancer immunity and the cancer-immune set point. Nature. 2017;541(7637):321-30.

27. Zhang Y, Chen L. Classification of advanced human cancers based on Tumor Immunity in the MicroEnvironment (TIME) for cancer immunotherapy. JAMA Oncol. 2016;2(11):1403-4. 
28. Murciano-Goroff YR, Warner AB, Wolchok JD. The future of cancer immunotherapy: microenvironment-targeting combinations. Cell Res. 2020;30(6):507-19.

29. Harrington KJ, Puzanov I, Hecht JR, et al. Clinical development of talimogene laherparepvec (T-VEC): a modified herpes simplex virus type-1-derived oncolytic immunotherapy. Expert Rev Anticancer Ther. 2015;15(12):1389-403.

30. Pan RY, Chung WH, Chu MT, et al. Recent development and clinical application of cancer vaccine: targeting neoantigens. J Immunol Res. 2018;2018:4325874.

31. Lin JR, Izar B, Wang S, et al. Highly multiplexed immunofluorescence imaging of human tissues and tumors using t-CyCIF and conventional optical microscopes. Elife. 2018;7.

32. Stack EC, Wang C, Roman KA, et al. Multiplexed immunohistochemistry, imaging, and quantitation: a review, with an assessment of Tyramide signal amplification, multispectral imaging and multiplex analysis. Methods. 2014;70(1):46-58.

33. Dixon AR, Bathany C, Tsuei M, et al. Recent developments in multiplexing techniques for immunohistochemistry. Expert Rev Mol Diagn. 2015;15(9):1171-86.

34. Walch A, Rauser S, Deininger SO, et al. MALDI imaging mass spectrometry for direct tissue analysis: a new frontier for molecular histology. Histochem Cell Biol. 2008;130(3):421-34.

35. Giesen C, Wang HA, Schapiro D, et al. Highly multiplexed imaging of tumor tissues with subcellular resolution by mass cytometry. Nat Methods. 2014;11(4):417-22.

36. Aichler M, Walch A. MALDI Imaging mass spectrometry: current frontiers and perspectives in pathology research and practice. Lab Invest. 2015;95(4):422-31.

37. Ahmed M, Broeckx G, Baggerman G, et al. Next-generation protein analysis in the pathology department. J Clin Pathol. 2020;73(1):1-6.

38. Angelo M, Bendall SC, Finck R, et al. Multiplexed ion beam imaging of human breast tumors. Nat Med. 2014;20(4):436-42.

39. Wang F, Flanagan J, Su N, et al. RNAscope: a novel in situ RNA analysis platform for formalin-fixed, paraffin-embedded tissues. J Mol Diagn. 2012;14(1):22-9.

40. Stahl PL, Salmen F, Vickovic S, et al. Visualization and analysis of gene expression in tissue sections by spatial transcriptomics. Science. 2016;353(6294):78-82.

41. Vickovic S, Eraslan G, Salmen F, et al. High-definition spatial transcriptomics for in situ tissue profiling. Nat Methods. 2019;16(10):987-90.

42. Merritt CR, Ong GT, Church SE, et al. Multiplex digital spatial profiling of proteins and RNA in fixed tissue. Nat Biotechnol. 2020;38(5):586-99.
43. Lee J, Geiss GK, Demirkan G, et al. Implementation of a multiplex and quantitative proteomics platform for assessing protein lysates using DNA-barcoded antibodies. Mol Cell Proteomics. 2018;17(6):1245-58.

44. Geiss GK, Bumgarner RE, Birditt B, et al. Direct multiplexed measurement of gene expression with color-coded probe pairs. Nat Biotechnol. 2008;26(3):317-25.

45. Beechem JM. High-plex spatially resolved rna and protein detection using digital spatial profiling: A technology designed for immuno-oncology biomarker discovery and translational research. Methods Mol Biol. 2020;2055:563-83.

46. Decalf J, Albert ML, Ziai J. New tools for pathology: a user's review of a highly multiplexed method for in situ analysis of protein and RNA expression in tissue. J Pathol. 2019;247(5): 650-61.

47. Toki MI, Merritt CR, Wong PF, et al. High-plex predictive marker discovery for melanoma immunotherapy-treated patients using digital spatial profiling. Clin Cancer Res. 2019;25(18): 5503-12.

48. Zugazagoitia J, Gupta S, Liu Y, et al. Biomarkers associated with beneficial PD-1 checkpoint blockade in Non-Small-Cell Lung Cancer (NSCLC) identified using high-plex digital spatial profiling. Clin Cancer Res. 2020.

49. Sautes-Fridman C, Petitprez F, Calderaro J, et al. Tertiary lymphoid structures in the era of cancer immunotherapy. Nat Rev Cancer. 2019;19(6):307-25.

50. Helmink BA, Reddy SM, Gao J, et al. B cells and tertiary lymphoid structures promote immunotherapy response. Nature. 2020;577(7791):549-55.

51. Amaria RN, Reddy SM, Tawbi HA, et al. Neoadjuvant immune checkpoint blockade in high-risk resectable melanoma. Nat Med. 2018;24(11):1649-54.

52. Rozeman EA, Prevoo W, Meier MAJ, et al. Phase Ib/II trial testing combined radiofrequency ablation and ipilimumab in uveal melanoma (SECIRA-UM). Melanoma Res. 2020;30(3): 252-60.

53. Farren MR, Sayegh L, Ware MB, et al. Immunologic alterations in the pancreatic cancer microenvironment of patients treated with neoadjuvant chemotherapy and radiotherapy. JCI. Insight. 2020;5(1).

54. Koelzer VH, Sirinukunwattana K, Rittscher J, et al. Precision immunoprofiling by image analysis and artificial intelligence. Virchows Arch. 2019;474(4):511-22.

55. Van TM, Blank CU. A user's perspective on GeoMxTM digital spatial profiling. Immuno-Oncol Technol. 2019;1:11-8.

Cite this article as: Wang N, Wang R, Zhang X, Li X, Liang Y \& Ding Z. Spatially-resolved proteomics and transcriptomics: An emerging digital spatial profiling approach for tumor microenvironment. Visualized Cancer Medicine. 2021; $2,1$. 